\title{
SOME RECENT DEVELOPMENTS IN THE THEORY \\ OF SPIRAL STRUCTURE
}

\author{
G. CONTOPOULOS \\ University of Thessaloniki, Greece
}

\section{Particle Resonance}

The particle resonance in a galaxy is of special interest because, according to the recent theory of Lynden-Bell and Kalnajs (1972), it provides the excitation of the density waves. In particular it is of interest to know if the density wave reaches the particle resonance, because the dispersion relation derived by Lin and Shu (1966) has a forbidden region if the parameter

$$
Q=\left(\left\langle\dot{r}^{2}\right\rangle /\left\langle\dot{r}^{2}\right\rangle_{\min }\right)^{1 / 2},
$$

where $\left\langle\dot{r}^{2}\right\rangle_{\min }^{1 / 2}=(0.2857)^{1 / 2} 2 \pi G \sigma_{0} / \kappa$ (with $\sigma_{0}$ the surface density and $\kappa$ the epicyclic frequency), is larger than one. If $Q=1$, Lin and Shu find two waves going through the resonance but the amplitude tends to infinity there.

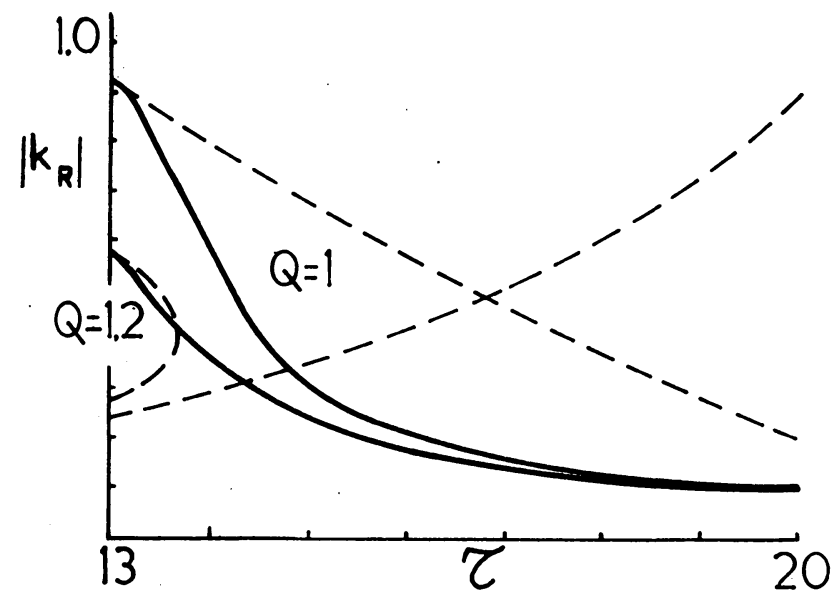

Fig. 1. The real part of the wave number in Schmidt's model of our Galaxy for $Q=1$ and $Q=1.2$ (-- Lin and Shu, _ our preliminary results). The particle resonance is assumed at $r=16.7 \mathrm{kpc}$.

We have derived a dispersion relation applicable near the particle resonance taking into account:

(1) the variation of the amplitude, considering the wave number $k$ as complex,

(2) the resonant stars, which give a pole in the response integral, and

(3) the fact that $k$ is relatively small in this region.

The preliminary dispersion relations for $Q=1$ and $Q=1.2$ are shown in Figure 1 .

We find only one wave going through the resonance, with group velocity directed always inwards. Near the resonance the wave number is small, and the corresponding 
waves are quite open. Finally we find only a small difference between trailing and leading waves.

\section{The Density Wave at the Centre of a Galaxy}

If there is no inner Lindblad resonance in a galaxy the density wave reaches the centre. A special treatment of this region is necessary because most orbits near the centre cannot be considered as epicycles. Mrs E. Athanassoula-Georgala (Athens) has studied this problem considering both elongated and epicyclic orbits. A dispersion relation valid near the centre is derived without the use of the asymptotic approximation.

\section{Trapping of Orbits around Barred Spirals}

M. Mihalodimitrakis (Thessaloniki) has extended the work of de Vaucouleurs and Freeman (1972) in studying the orbits around barred spirals. He considered two models of barred spirals: (a) a homogeneous ellipsoid and (b) a homogeneous parallelepiped, while de Vaucouleurs and Freeman considered an inhomogeneous ellipsoid. Many cases were studied with various values of the axis-ratios and of the rotational velocity.

Three families of periodic orbits surrounding the bar were found, two of them direct and one retrograde. One of the two direct families is new (Figure 2). A large set of non-periodic orbits is trapped in rings around the bar.

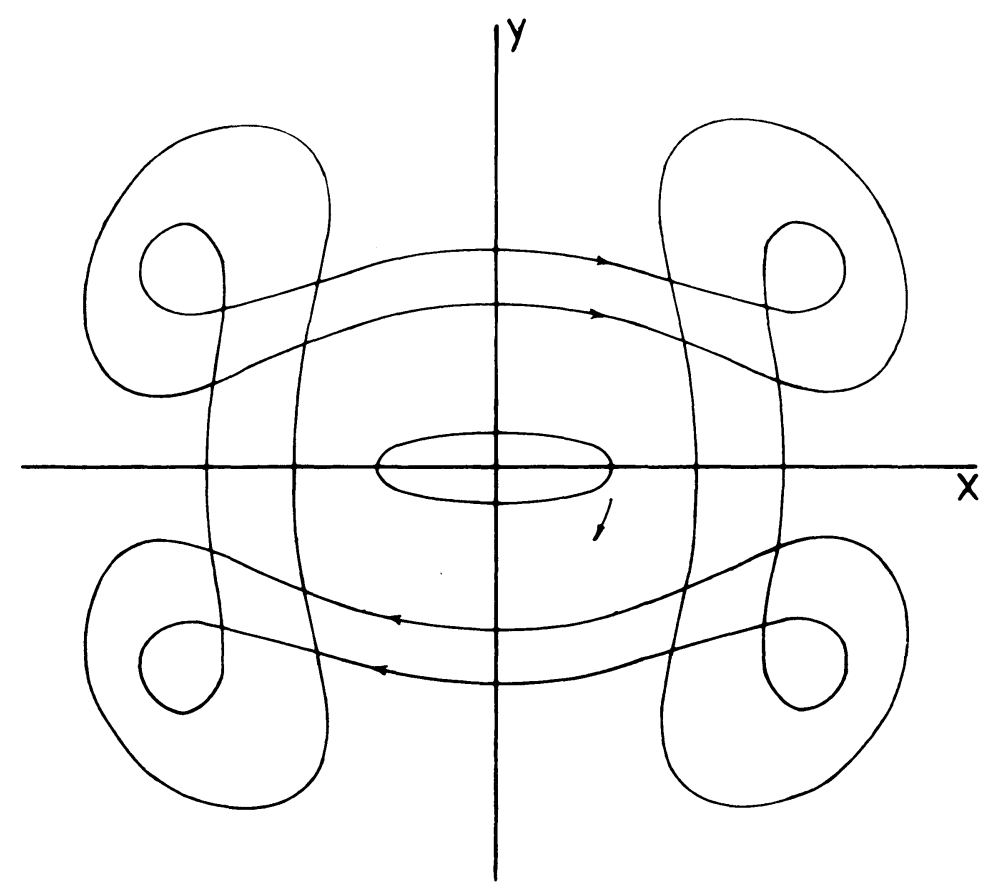

Fig. 2. A new family of periodic orbits around a barred spiral. 
Several periodic orbits were also found around the Lagrangian points $L_{4}, L_{5}$, perpendicular to the axis of the bar, many of them stable.

The Lagrangian points $L_{1}, L_{2}$ on the axis of the bar are always unstable in the case of the ellipsoid but they are stable in some cases of the parallelepiped model.

A theory to describe the trapping of orbits around the (stable) Lagrangian points is developed, similar to the theory of the Lagrangian points $L_{4}, L_{5}$ of a regular spiral galaxy (Contopoulos, 1973).

\section{Quasi-Linear Theory}

A quasi-linear theory of density waves has been developed by Mrs E. Dekker (Leiden), extending the work of Lynden-Bell and Kalnajs (1972).

The growth coefficient $\gamma$ is derived from the equation

$$
2 \gamma=\dot{E} / E
$$

where $E$ is the rate of energy change of the wave due to resonant stars, while $E$ is the energy stored in non-resonant stars. One immediate application is that, if there is no inner Lindblad resonance, $\gamma$ is positive, i.e. the wave grows.

The growth of the wave affects the zero-order distribution function $f_{0}$. As the amplitude of the wave increases, $f_{0}$ changes until a stationary finite wave is reached. In this research the usual methods of the quasi-linear theory of plasma waves are applied.

This research was supported in part by the Greek National Research Foundation.

\section{References}

Contopoulos, G.: 1973, Astrophys. J. 181, 657.

de Vaucouleurs, G. and Freeman, K. C.: 1972, Vistas in Astronomy 14, 16 j.

Lin, C. C. and Shu, F. H.: 1966, Proc. Nat. Acad. Sci. 55, 229.

Lynden-Bell, D. and Kalnajs, A. J.: 1972, Monthly Notices Roy. Astron. Soc. 157, 1.

\section{DISCUSSION}

G. de Vaucouleurs: Does the study of a bar relate to a bar alone or a bar in a disk?

Contopoulos: A bar alone.

Heidmann: With respect to the Lin and Shu theory we have $21-\mathrm{cm}$ line observations of lenticular galaxies which might be of interest. We found that lenticulars have neutral hydrogen components comparable in mass and extent to those of Sa galaxies (Astron. Astrophys. 21, 303, 1973). This then raises the question as to why lenticulars have no spiral arms but Sa's have.

Lindblad: Is anything known about the rotation curves of the lenticular galaxies you mentioned?

Heidmann: Normal rotation curves were obtained, for instance for NGC 3115, by optical astronomers. For our best case, NGC 5102, the width of the $21-\mathrm{cm}$ line is compatible with a normal rotation.

Wilson: What growth times does Mrs Dekker get for resonances with the epicyclic orbits?

Contopoulos: She has no numerical results yet. However, Kalnajs (IAU Symp. 38, 318, 1970) found numerically a mode for a model of M31 with a growth time of a few $\times 10^{8} \mathrm{yr}$.

Wilson: I have done a similar linear calculation giving instabilities in the galactic gas of $1 \mathrm{kpc}$ wavelength and with a growth time of about $4 \times 10^{8} \mathrm{yr}$. 